\title{
Molecular docking and binding energy studies on nuraminidase of h1n1 reveal possible answer to its resistance for oseltamivir
}

\author{
Meshram R.J.* and Jangle S.N. \\ ${ }^{\star}$ Center for Biotechnology, Pravara Institute of Medical Sciences, Loni, MS, India, \\ E.Mail: rohan_meshram@rediffmail.com, biotechnology@pmtpims.org, 09822725648
}

\begin{abstract}
Neuraminidase (NA) is the enzyme coded by Influenza- $A$ virus genome that catalyzes the removal of terminal sialic acid residues from viral and cellular glycoconjugates. It cleaves off the terminal sialic acids on the glycosylated NA during virus budding to facilitate virus release, making it most important target for designing drug against Flu. Recently cases are reported of Influenza virus becoming resistant to NA inhibitors like Oseltamivir. The outbreak of Swine flu and its resistance to oseltamivir is suspected to be caused due to mutation $\mathrm{H} 274 \mathrm{Y}$ in the enzyme neuraminidase of $\mathrm{H} 1 \mathrm{~N} 1$ strain of influenza virus. This work involved active site analysis, molecular docking and binding energy studies on NA that demonstrate the conformational changes in active site which might result in increase in binding energy of oseltamivir when it is mutated as $\mathrm{H} 274 \mathrm{Y}$.

Keywords- Universal Force Field, Parametric Method 3, ArgusLab, AScore Scoring Function, Binding Affinity

\section{Introduction}

WHO report on Pandemic H1N1 2009 activity in India caused 12,787 cumulative confirmed cases and 413 cumulative deaths cases as on 18 oct 2009 fig. (1) and fig. (2). A search in Therapeutic Target Database [1] identified two successful targets against influenza $\mathrm{H} 1 \mathrm{~N} 1$, those include Influenza $A$ virus $M 2$ protein and neuraminidase, while targets like RNA-dependent RNA polymerase, macrophage inflammatory protein-2-

Simplified Molecular Input Line Entry System (SMILES) [9] notation of drug was obtained from Drugbank [10] database; mol file of drug was generated using online server "Online Smiles Translator and Structure File Generator". ArgusLab 4.0.1 was employed for further ligand preparatory steps, docking calculation and initial addition of hydrogen and cleaning the geometry of target molecule.
\end{abstract} alpha and hemagglutinin are under clinical trial. CAP-dependent endonuclease is one of research target. Newer and efficient drugs against H1N1 are thus very essential to improve the still worsening situation in India. Oseltamivir and zanamivir are two existing drugs used against Influenza $\mathrm{H} 1 \mathrm{~N} 1$ infection in pandemic [2]. Oseltamivir is an antiviral drug, a neuraminidase inhibitor used in the treatment and prophylaxis of both influenza A and influenza B [3, 4]. Oseltamivir is a prodrug (usually administered as phosphate) specifically, an ethyl ester prodrug requiring ester hydrolysis for conversion to the active form, oseltamivir carboxylate [5]. The proposed mechanism of action of oseltamivir and zanamivir is inhibition of influenza virus neuraminidase with the possibility of alteration of virus particle aggregation and release as both zanamivir and oseltamivir acts as a transitionstate analogue inhibitor of influenza neuraminidase [6].

\section{Methodology}

Structural analysis including structural alignment, calculation of Root Mean Square Deviation (RMSD) and determination and analysis of active site was carried out using SwissPDB Viewer Version 3.7 (SPDBV 3.7) [7,8], while steps in target preparation like removal of solvent molecules and removal of other unnecessary components like cofactors were completed using SPDBV 3.7. In process of "ligand preparation"

\section{Target Protein Acquisition}

Protein targets crystallized with and without ligand were downloaded from database Protein Data Bank (PDB) [11]. Mutated and unmutated targets were also downloaded from the PDB. 2HTY AND 2HU4 are NA models crystallized with oseltamivir and model 3B7E is a NA crystallized with zanamivir. $3 \mathrm{CKZ}$ is a model mutated as $\mathrm{H} 274 \mathrm{Y}$.

\section{Structural Analysis of Target Proteins}

After structure comparison, by structure alignment between two unmutated models, one crystallized with oseltamivir (2HU4) and other crystallized with zanamivir (3B7E) with help of superimposition algorithm, the RMSD was calculated to find out structural similarity between two unmutated models. The most common parameter that expresses the difference between two protein structures is RMSD, or Root mean squared deviation, in atomic positions between the two structures. Protein structures are described in cartesian coordinates. RMSD is a function of the distance between atoms in one structure and the same atoms in another structure. Thus, if one molecule starts out in a different position with respect to the other molecule in the reference coordinate system, the RMSD between the two molecules will be large. RMSD was computed in terms of all the atoms in a protein backbone and the value were found to 
be very low as 0.40 Angstrom signifying very close structural similarity. Table 1 and fig. (4) shows the relative distance of aminoacid residues from oseltamivir and zanamivir in models $2 \mathrm{HU} 4$ and $3 \mathrm{~B} 7 \mathrm{E}$. It clarifies that all the amino acid residues in 5 Angstrom area of zanamivir which interact with it through their side chains remain at same position in NA, it don't matter if it is bounded by zanamivir or oseltamivir. These facts suggest that binding of oseltamivir do not impart any significant conformational changes in the target as compared to binding of zanamivir. Hence models crystallized with zanamivir can be utilized to study effect of oseltamivir on the same target. When NA is mutated at $274^{\text {th }}$ position and histidine is replaced by tyrosine, the tyrosine being more bulky than histidine approach forward occupying more area in active site. This might create conformational changes which result in increase in binding energy fig. (5).

\section{Processing of Target Proteins}

PDB models $2 \mathrm{HTY}$ and $3 \mathrm{CKZ}$ were used for docking and binding energy calculation studies. The quaternary structure of NA is build up as a tetramer; model 2HTY contains two copies of the NA, while $3 \mathrm{CKZ}$ is a monomer. Monomer copy of NA from $3 C K Z$ model was obtained by removing the other 7 copies using SPDBV Select and Build utilities. Both the Models contained solvent molecules along with hetero groups like its inhibitors (zanamivir and oseltamivir) and few of the calcium ions. Solvent and heterogroups were also removed using SPDBV Select and Build utilities. Addition of Hydrogen was based on the hybridization and current bonding pattern of the heavy atoms in molecule and further cleaning process was done utilizing forcefield based approaches using Universal Force Field (UFF) [12-14], all included in the Arguslab software.

\section{Determination of active site}

The active site was defined from the coordinates of the ligand in the original PDB files $3 C K Z$ for the receptor protein. Residues which lie within 5 Angstrom unit area of ligand that interact with it through their side chain were identified and were considered as Active site residues. Out of these residues TRP 178 do not contribute its side chain to active site; ARG 224 forms a Non Conventional $\mathrm{H}$ bond with the ligand. TYR 274 side chain does not contribute its side chain to active site but it forms hydrogen bond with side chain of ARG 224 stabilizing the active site (Fig. 6 A). Finally, nine residues, including ARG 118, ASP 151, ARG 152, GLU 227, GLU 276, ARG 292, ARG 371, TRP 178, and ARG 224 were determined as to be active site in both the models (fig. $6 \mathrm{~B}$ ).

\section{Preparation of Ligands}

Oseltamivir SMILES was obtained from Drugbank database and was further translated to mol file using "On Line SMILES Translator and Structure File Generator" utility which relies on CACTVS technology and utilizes the algorithm of program COoRdINAtes (CORINA) [15- 17] to generate 3D atomic coordinates of a molecule. A Molecular Mechanics (MM) method UFF was used for refining initial geometries, using the "Clean Geometry" option in the ArgusLab Software. Hydrogens were added using "Add Hydrogens" option. "Clean Hybridization" option was utilized to check all the bond types and set proper ones if some went wrong. Final Geometry optimization was performed using a semiempirical Quantum Mechanic (QM) method Parametric Method 3 (PM3) [18-24]. The Initial geometry refinement using UFF force field and final Geometry optimization using PM3 procedures were repeated till the Optimizations Converge. Finally the oseltamivir model was saved as a mol file for further docking procedure.

\section{Docking}

The docking between receptor and ligand was performed using the "Dock a ligand" option. Nine residues that lie within 5 Angstrom unit area of ligand that interacts with it through their side chain were considered as Active site residues. A spacing of $0.4 \AA$ between the grid points was used. Docking simulations were performed by selecting "ArgusDock" as the docking engine. An exhaustive Search was performed by enabling "High precision" option in Docking precision menu, "Dock" was chosen as the calculation type, "flexible" for the ligand and the AScore was used as the scoring function. At maximum 150 poses were allowed to be analyzed, binding site box size was set to $20 \times 20 \times 20$ angstroms so as to encompass the entire active site. The AScore function, with the parameters read from the AScore.prm file was used to calculate the binding energies of the resulting docked structures. This file contains the coefficients for each term in the scoring function. Each docking run was repeated five times to get best results. The docking results are described in Table 2. The resulting docking molecules were saved as pdb files and Molecular graphics images were reproduced using the UCSF Chimera software [25] fig. (7).

\section{Results and Discussion}

The field of molecular docking has emerged during the last three decades and now is becoming an integral aspect in drug discovery and development area. Molecular docking is utilized for the prediction of protein-ligand complexes which is composed of two components: a search algorithm, an algorithm that creates possible protein-ligand complex geometries, and thus performs the process of 
"pose generation" and a scoring function that predicts the binding affinity of the ligand to the protein based on the complex geometry. ArgusLab provides the stochastic search, analogous to the genetic algorithm normally used as a standard in Molecular docking studies, along with this an exhaustive search method based on identification of complementary shapes of the ligand and the receptor, referred to as "ShapeDock" or "ArgusDock" is also utilized. The docking results are described in Table 2. From the analysis of the values in Table 2. it is evident that the binding energy of mutated complex is more than that of unmutated complex. There is increase in binding energy by approximately 420 $\mathrm{Kcal} /$ mole on transpiration of mutation. Binding energies are most widely used mode of measuring binding affinity of a ligand. Thus increase in binding energy due to mutation might decrease the binding affinity of oseltamivir towards NA, making virus resistant to NA inhibitors.

\section{Conclusion}

Here we propose an idea that difference in binding energies might arise due to mutations which can cause conformational changes that affect the binding affinity of a ligand, as it is known that binding energies reflects the binding affinity . However, along with binding energies, several other physical effects like electrostatics, van der waals forces, hydrogen bonding, and hydrophobic and entropic effects influence the binding affinity; these are also needed to be evaluated for calculating binding affinity of ligands or drug candidates. We have also compared the structures of NA crystallized with oseltamivir and zanamivir and shown that active site remains the same but when mutated as $\mathrm{H} 274 \mathrm{Y}$, the tyrosine step forward in active site. New drugs can be designed or existing drugs can be modified based on its interaction with new tyrosine residues.

\section{References}

[1] Chen X., Ji Z.L., Chen Y.Z. (2002) Nucleic Acids Research, 30(1), 412-5.

[2] Ward P., Small I., Smith J., Suter P., Dutkowski R. (2005) The Journal of Antimicrobial Chemotherapy, 55 (1), i5-i21.

[3] Imming P., Sinning C., Meyer A. (2006) Nature Reviews Drug Discovery, 5(10), 821-34.

[4] Overington J.P., Al-Lazikani B., Hopkins A.L. (2006) Nature Reviews Drug Discovery, 5(12), 993-6.
[5] Meindl P., Bodo G., Palese P., Schulman J., Tuppy H. (1974) Virology, 58(2), 457-63.

[6] Wang M.Z., Tai C.Y., Mendel D.B. (2002) Antimicrobial Agents Chemotherapy, 46(12), 3809-16.

[7] Boeckmann B., Bairoch A., Apweiler R., Blatter M.C., Estreicher A., Gasteiger .E., Martin .M.J., Michoud.K., O'Donovan. C., Phan I., Pilbout S., Schneider M. (2003) Nucleic Acids Research, 31, 365-370.

[8] Guex N., Peitsch M.C. (1997) Electrophoresis, 18, 2714-2723.

[9] Weininger D. (1988) Journal of Chemical Information and Computer Sciences, 28(1), 31-36.

[10] Wishart D.S., Knox C., Guo A.C., Shrivastava S., Hassanali M. (2003) Nucleic Acids Research 34, 668-672.

[11] Berman H.M., Westbrook J., Feng Z., Gilliland G., Bhat T.N., Weissig H., Shindyalov I.N., Bourne P.E. (2000) Nucleic Acids Research, 28, 235-242

[12] [Rappe A.K., Casewit C. J., Colwell K. S., Goddard III W.A., Skiff W. M. (1992) The Journal of the American Chemical Society, 114, 10024-10035.

[13] Casewit C. J., Colwell K. S., Rappe A. K. (1992) Journal of the American Chemical Society, 114, 10035-10046.

[14] Rappe A. K., Colwell K. S., Casewit C. J. (1993) Inorganic Chemistry, 32, 3438-3450.

[15] Sadowski J., Rudolph C., Gasteiger J. (1992) Analytica Chimica Acta, 265, 233-241.

[16] Sadowski J., Gasteiger J., Klebe G. (1994) The Journal of Chemical Information and Modeling, 34, 1000-1008.

[17] Schönberger H., Schwab C.H., Hirsch A, Gasteiger, J. (2000) The Journal of Molecular Modeling, 6, 379-395.

[18] Thompson M.A., Zerner M. C. (1991) Journal of the American Chemical Society, 113, 8210.

[19] Thompson M.A., Glendening E.D., Feller D. (1994) Journal of Physical Chemistry, 98, 10465-10476.

[20] Thompson M.A., Schenter G.K. (1995) Journal of Physical Chemistry, 99, 6374-6386.

[21] Thompson M.A. (1996) Journal of Physical Chemistry, 100, 14492-14507.

[22] James J., Stewart P. (1989) The Journal of Computational Chemistry, 10, 209-220

[23] James J., Stewart P. (1989) The Journal of Computational Chemistry, 10, 221-264.

[24] Gasteiger J., Rudolph. C., Sadowski J. (1990) Tetrahedron Computer Methodology, 3, 537547.

[25] Pettersen E.F., Goddard T.D., Huang C.C., Couch G.S., Greenblatt D.M., Meng E.C., Ferrin T.E. (2004) Journal of Computational Chemistry, 25(13), 1605-1612. 


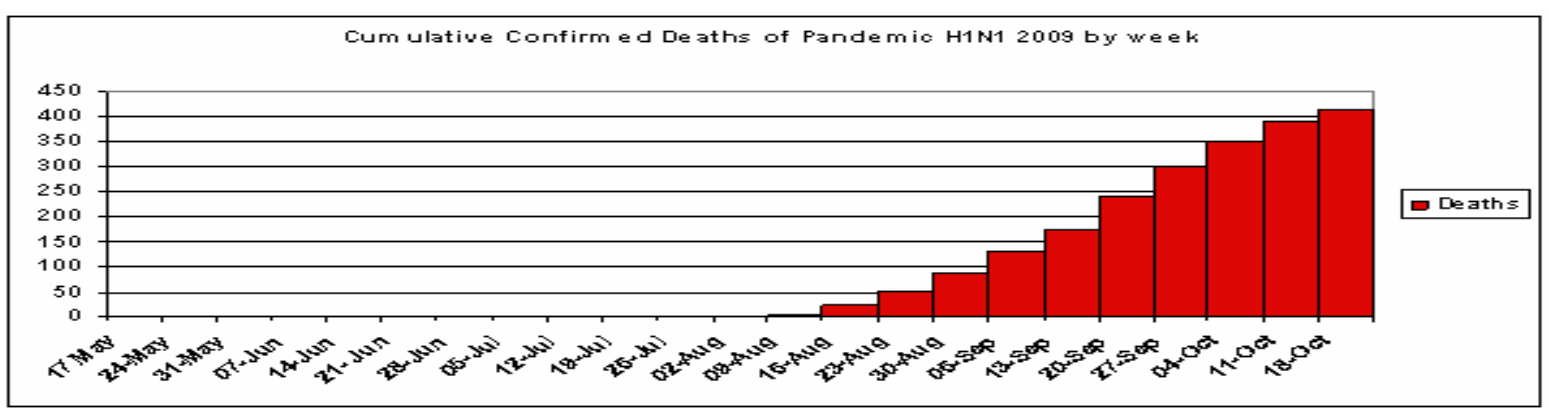

Fig. 1- Cumulative confirmed deaths of pandemic H1N1 2009 by week

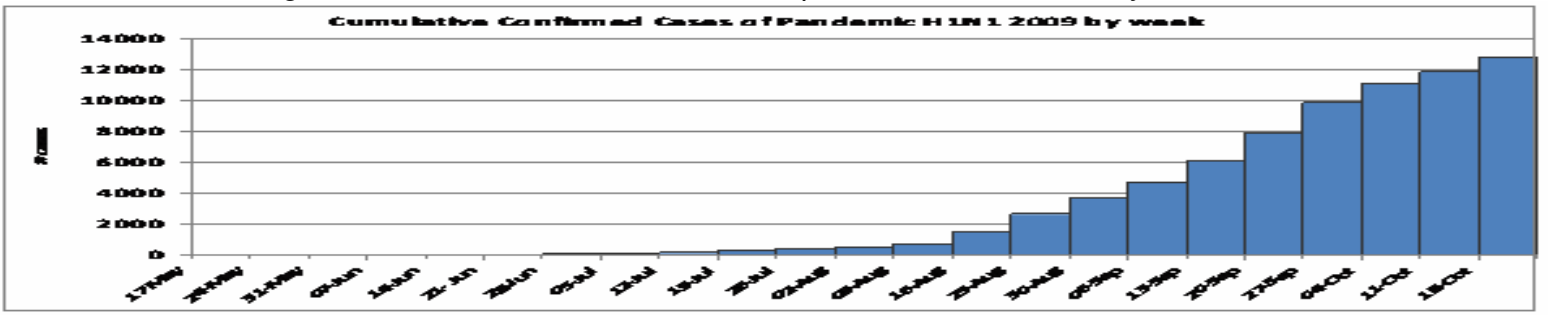

Fig. 2- Cumulative confirmed cases of pandemic H1N1 2009 by week

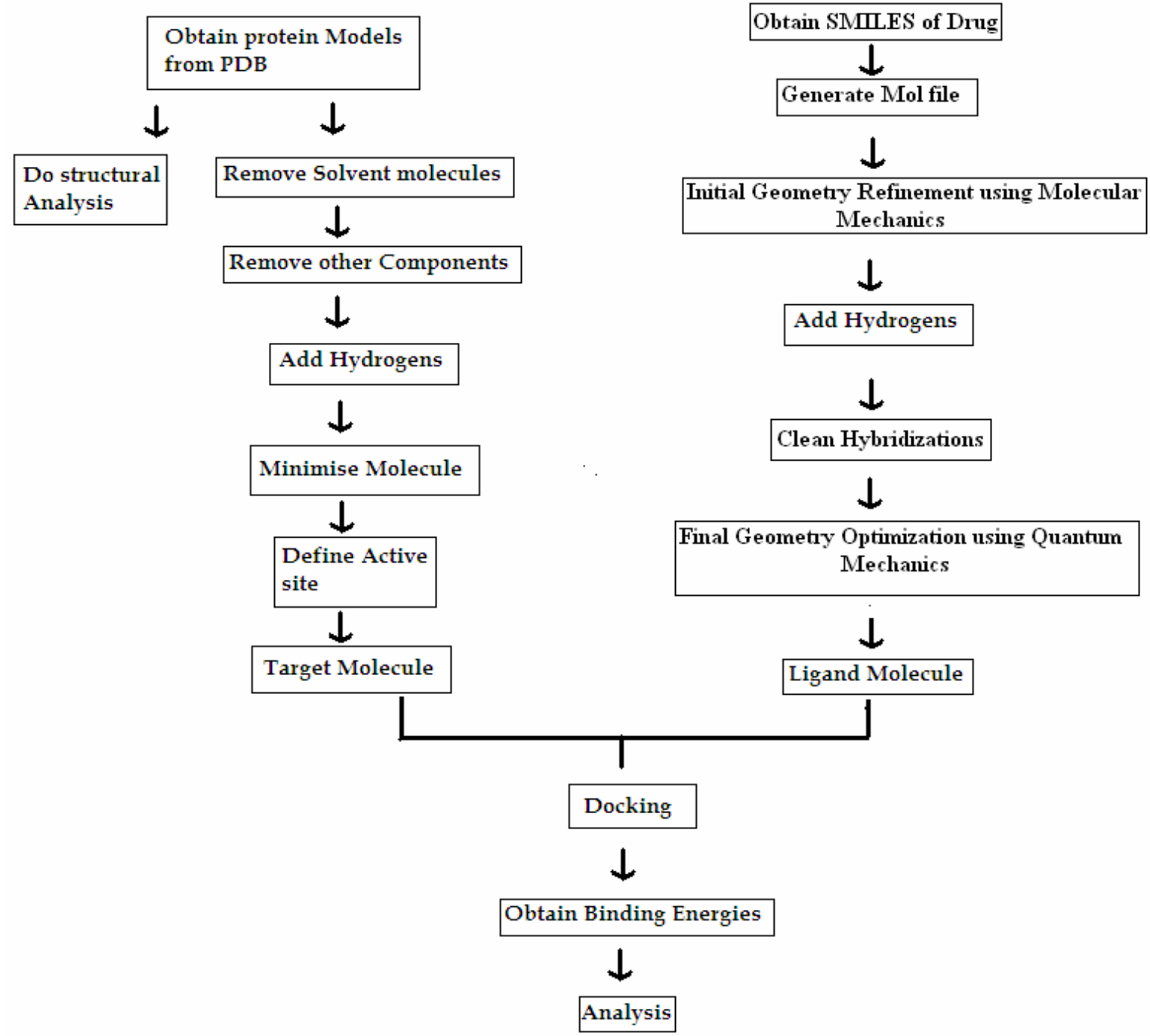

Fig. 3- Methodology applied in present work 


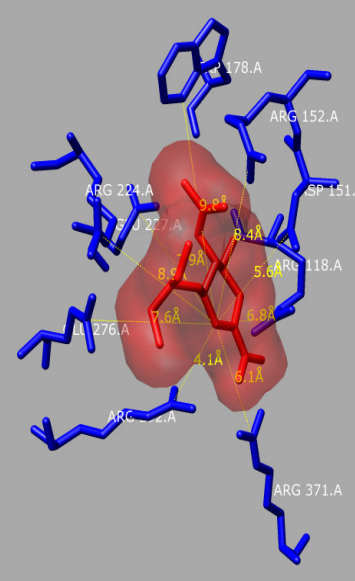

Fig. 4-A- Distances of active site residues from Oseltamivir (Red)

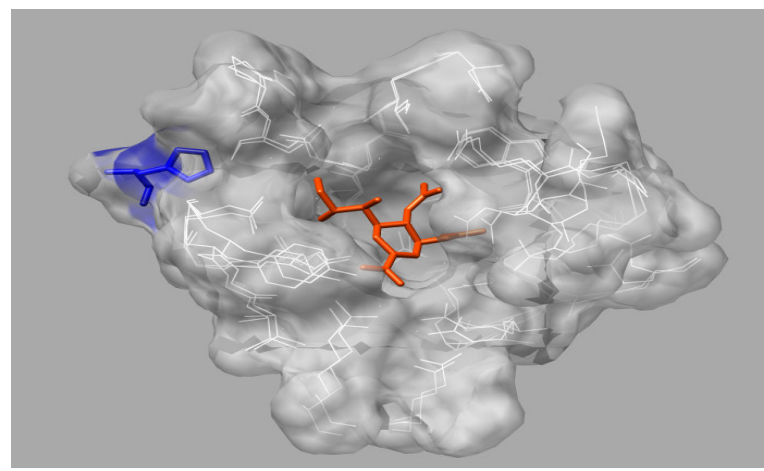

Fig. 5-A- Active site of NA before mutation with Histidine (Blue) at 274 position and zanamivir Orange

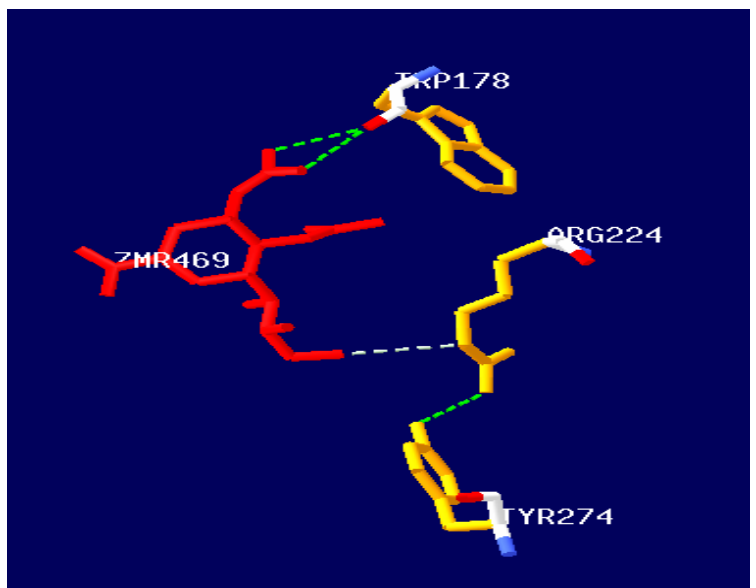

Fig. 6- A- Interaction of zanamivir (Red) with Tryptophan 178 backbone (CPK) and nonconventional Hydrogen bond (Grey) with side chain of Arginine 224 (Yellow) along with relative position of Tyrosine 274 and its interaction through Hydrogen bond with Arginine 224

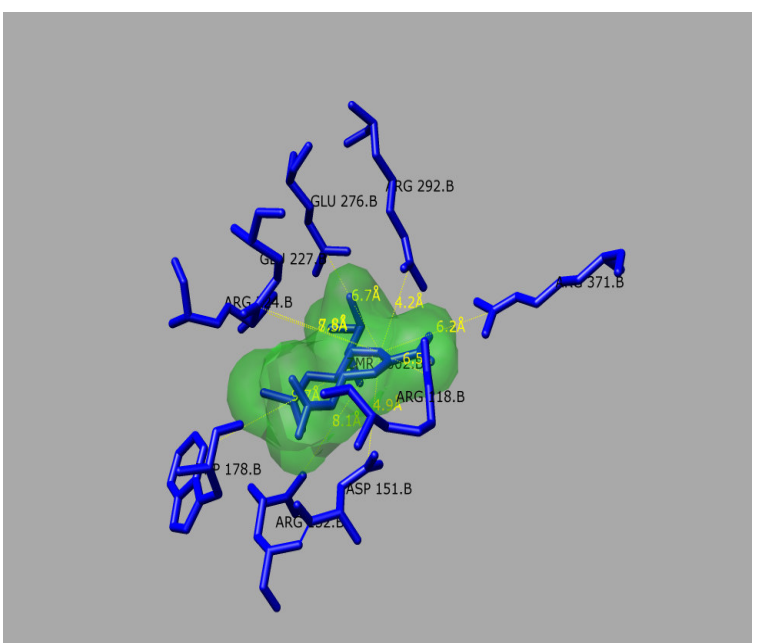

Fig. 4-B- Distances of active site residues from zanamivir (green)

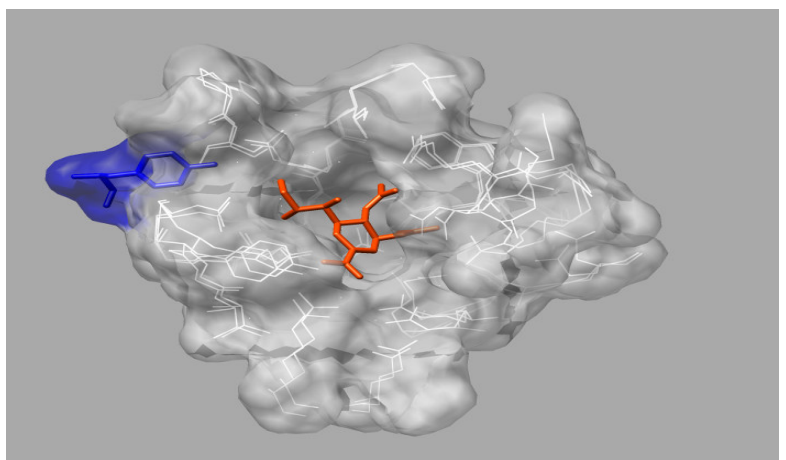

Fig. 5-B- Active site of NA after mutation with Tyrosine (Blue) at 274 position and zanamivir Orange

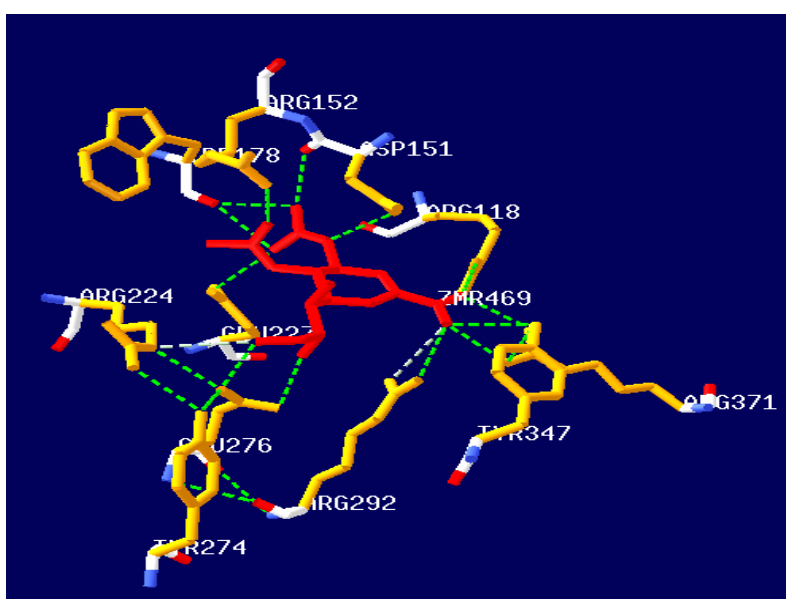

Fig. 6-B: zanamivir (Red) in Active site interacting with other residue's side chains (Yellow) and backbone (CPK) 


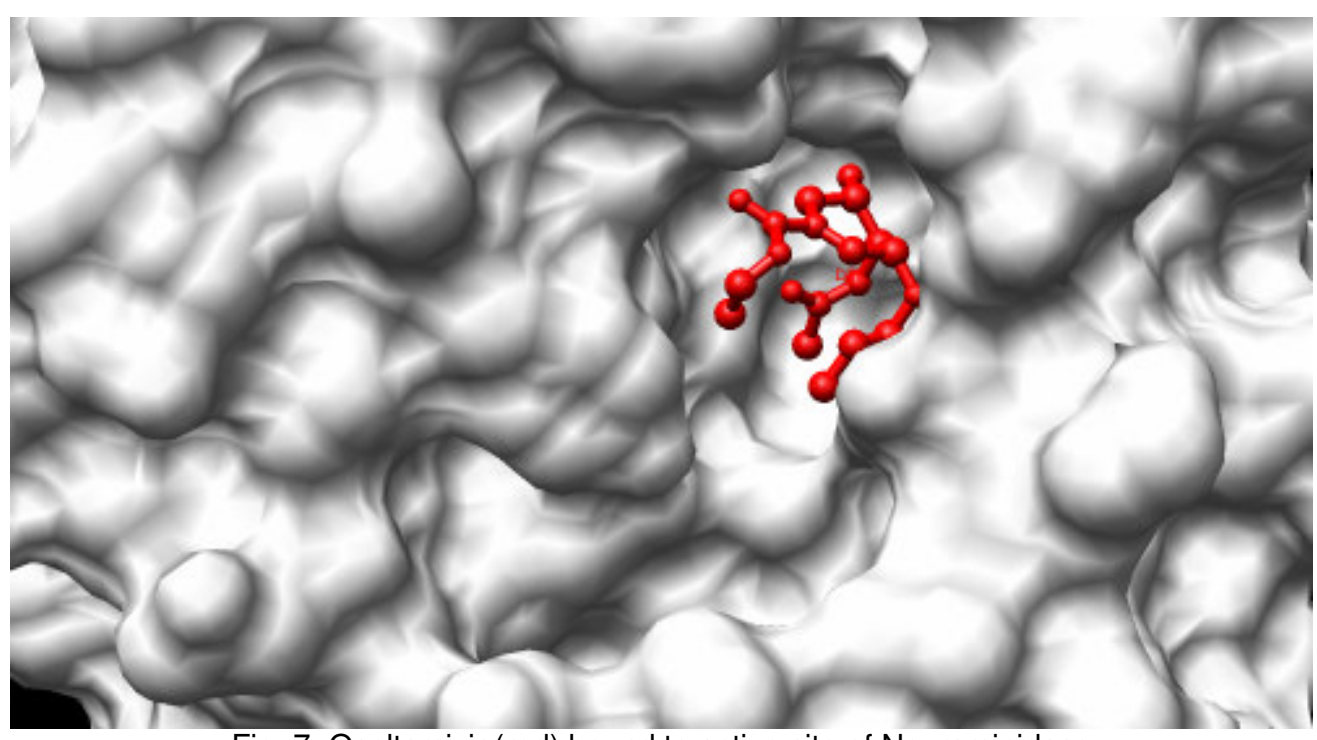

Fig. 7- Oseltamivir (red) bound to active site of Neuraminidase

Table 1- The relative distance of active site amino acid residues from oseltamivir and zanamivir

\begin{tabular}{|l|l|l|l|l|l|l|l|l|l|}
\hline \multirow{3}{*}{ Ligands } & \multicolumn{9}{|l|}{ Active site Residues along with distance from ligand in Angstrom units } \\
\cline { 2 - 11 } & $\begin{array}{l}\text { ARG } \\
118 \mathrm{CZ}\end{array}$ & $\begin{array}{l}\text { ASP } \\
151 \mathrm{CG}\end{array}$ & $\begin{array}{l}\text { ARG } \\
152 \mathrm{CZ}\end{array}$ & $\begin{array}{l}\text { GLU } \\
227 \mathrm{CD}\end{array}$ & $\begin{array}{l}\text { GLU } \\
276 \mathrm{CD}\end{array}$ & $\begin{array}{l}\text { ARG } \\
292 \mathrm{CZ}\end{array}$ & $\begin{array}{l}\text { ARG } \\
371 \mathrm{CZ}\end{array}$ & $\begin{array}{l}\text { TRP } 178 \\
\text { CE3 }\end{array}$ & $\begin{array}{l}\text { ARG } \\
224 \mathrm{CZ}\end{array}$ \\
\hline $\begin{array}{l}\text { C7 of } \\
\text { oseltamivir }\end{array}$ & 6.79 & 5.56 & 8.41 & 7.94 & 7.58 & 4.13 & 6.12 & 9.83 & 8.87 \\
\hline O6 of zanamivir & 6.54 & 4.94 & 8.17 & 7.88 & 6.66 & 4.17 & 6.24 & 9.70 & 8.43 \\
\hline
\end{tabular}

Table 2- Energy Values obtained in Docking Calculations

\begin{tabular}{|c|c|c|c|c|c|c|c|c|c|c|c|c|}
\hline \multirow[b]{2}{*}{$\begin{array}{l}\text { Target } \\
\text { status }\end{array}$} & \multicolumn{9}{|c|}{ Energy values in Active site residues in $\mathrm{Kcal} / \mathrm{mol}$} & \multirow{2}{*}{$\begin{array}{l}\text { AVG } \\
\text { ENERGY } \\
\text { VALUES IN } \\
\text { KCAL/MOL } \\
\text { E }\end{array}$} & \multirow{2}{*}{$\begin{array}{l}\text { AVG } \\
\text { ENERGY } \\
\text { VALUES } \\
\text { IN } \\
\text { CAL/MO } \\
\text { LE } \\
\end{array}$} & \multirow{2}{*}{$\begin{array}{l}\text { DIFF } \\
\text { ERE } \\
\text { NCE } \\
\text { IN } \\
\text { CAL/ } \\
\text { MOL } \\
\text { E }\end{array}$} \\
\hline & $\begin{array}{l}\text { ARG } \\
118\end{array}$ & $\begin{array}{l}\text { ASP } \\
151\end{array}$ & $\begin{array}{l}\text { ARG } \\
152\end{array}$ & $\begin{array}{l}\text { GLU } \\
227\end{array}$ & $\begin{array}{l}\text { GLU } \\
276\end{array}$ & $\begin{array}{l}\text { ARG } \\
292\end{array}$ & $\begin{array}{l}\text { ARG } \\
371\end{array}$ & $\begin{array}{l}\text { TRP } \\
178\end{array}$ & $\begin{array}{l}\text { ARG } \\
224\end{array}$ & & & \\
\hline $\begin{array}{l}\text { Normal } \\
2 \mathrm{HTY}\end{array}$ & 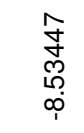 & 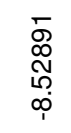 & $\begin{array}{l}0 \\
\stackrel{0}{0} \\
\infty \\
\infty \\
\stackrel{0}{1}\end{array}$ & 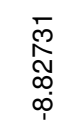 & 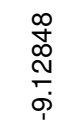 & $\begin{array}{l}\stackrel{0}{0} \\
\stackrel{\infty}{\$} \\
\stackrel{N}{\uparrow} \\
\end{array}$ & 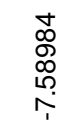 & 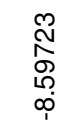 & 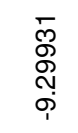 & -8.35382 & -8353.82 & \multirow{2}{*}{$\begin{array}{l}419 . \\
8722\end{array}$} \\
\hline $\begin{array}{l}\text { Mutated } \\
\text { 3CKZ }\end{array}$ & 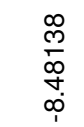 & 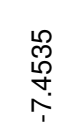 & 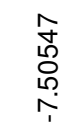 & $\begin{array}{l}\text { वे. } \\
\text { ల్ల } \\
\infty \\
\text { i. }\end{array}$ & 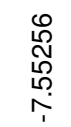 & $\begin{array}{l}\frac{\Gamma}{\infty} \\
\stackrel{N}{N} \\
\stackrel{1}{i}\end{array}$ & 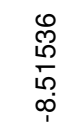 & \begin{tabular}{l}
$\hat{N}$ \\
\multirow{0}{0}{} \\
\multirow{N}{N}{} \\
\end{tabular} & 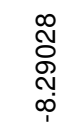 & -7.93395 & -7933.95 & \\
\hline
\end{tabular}

